\title{
Wavy cracks in drying colloidal films
}

\author{
Lucas Goehring, $\dagger^{a b c}$ William J. Clegg ${ }^{a}$ and Alexander F. Routh ${ }^{* b c}$ \\ Received 26th May 2011, Accepted 19th July 2011 \\ DOI: $10.1039 / \mathrm{c} 1 \mathrm{sm05979c}$
}

Fracture mechanics successfully predicts when cracks will grow. Describing the path that cracks follow, however, has remained difficult. The study of crack paths has recently focused on a single experimental system, that of thermally quenched glass, where straight, wavy, helical, and branched cracks appear under different conditions. Several models of crack path prediction have been developed but none is generally accepted. Here we show that slowly oscillating wavy cracks can form during the drying of a colloidal dispersion. These drying films are subject to large stress gradients perpendicular to the mean direction of crack growth. Under these conditions existing models do not predict periodic paths. We show, instead, how to model crack paths by allowing a growing crack to curve towards the direction of maximum energy release rate. Not only does this explain wavy cracks in drying films, and correctly describe the wavelength dependence of our experiments, but it is generally applicable to predicting crack paths in spatially varying stress fields.

Prediction of the conditions under which a crack can grow, in a stressed body, are given by the fracture mechanics developed by Griffith and Irwin. ${ }^{1}$ It follows from these theories that a crack in an isotropic medium should also tend to grow in the direction of some maximum strain energy release rate. However, the related 'principle of local symmetry' has instead been most frequently invoked to predict how cracks grow. ${ }^{2}$ This requires cracks to follow paths along which the shear stress in the immediate vicinity of the crack tip is always zero. This principle has been developed alongside experiments on quenched glass, where straight, wavy, helical, and branched cracks appear under different conditions..$^{3-8}$ Recently, however, these experiments have found that local symmetry is violated. ${ }^{8}$ Here we show that drying colloidal dispersions can also display wavy cracks. We find that local symmetry does not predict periodic crack paths in drying films, but that a model, based on a crack seeking to maximise its energy release rate, does. This work touches on the fundamental

${ }^{a}$ Department of Materials Science and Metallurgy, University of Cambridge, Pembroke Street, Cambridge, UK CB2 $3 Q Z$

${ }^{b}$ Department of Chemical Engineering and Biotechnology, University of Cambridge, Pembroke Street, Cambridge, UK CB2 $3 R A$

${ }^{c} B P$ Institute for Multiphase Flow, University of Cambridge, Madingley Rise, Madingley Road, Cambridge, UK CB3 OEZ

$\dagger$ Present Address: Max Planck Institute for Dynamics and Self-Organization, Bunsenstraße 10, 37073 Göttingen, Germany. question of the path taken by a growing crack, and suggests how to construct a general crack path model.

Films, of either colloidal latex (prepared as described elsewhere ${ }^{9}$ ) or silica (Ludox TM-40, HSA, or FM), were spread onto level glass slides, and allowed to dry by evaporation from the exposed upper surface. The films dried directionally, solidifying, and cracking, from the edges inward, ${ }^{10}$ as outlined in Fig. 1(a), with final film thicknesses of order $10 \mu \mathrm{m}$. In addition to the expected array of parallel straight cracks, ${ }^{10,11}$ cracks were also seen to advance along wavy, or oscillatory, paths. The tips of the straight cracks lay near a common fracture front, and, as shown in Fig. 1(a), small displacements away from this line do not appear to affect the crack pattern. Wavy cracks, however, always lagged well behind their neighbours, typically by many times the average crack spacing, such that each wavy crack was bounded by two pre-existing, parallel cracks, as shown in Fig. 1(b). The crack paths were observed in dry films with a digital microscope, and the
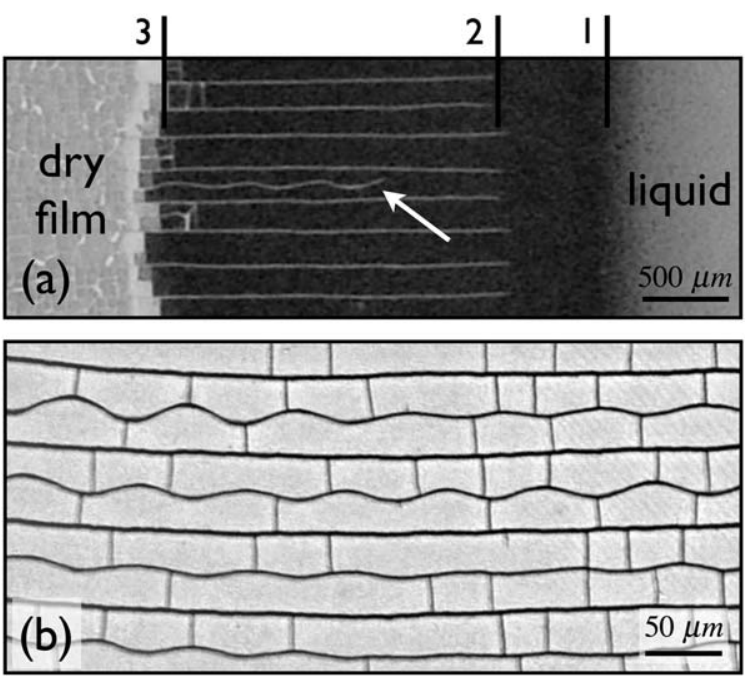

Fig. 1 Wavy cracks in drying colloidal dispersions. (a) During directional drying, a film solidifies from its edges, inwards. A series of coparallel drying fronts form, and typically advance at speeds of order $1 \mu \mathrm{m} \mathrm{s}^{-1}$. Initially (1) the dispersion solidifies into a rigid particle raft, although the pore spaces between particles remain filled with fluid. Capillary forces build up in the rigid film, as it wicks liquid to replace that lost to evaporation. These pressures can drive (2) fracture, and (3) the draining of the interstitial pores. Wavy cracks were seen to advance behind the common fracture front. (b) When drying is complete, each wavy crack is bounded on either side by a straight crack. 
(a)

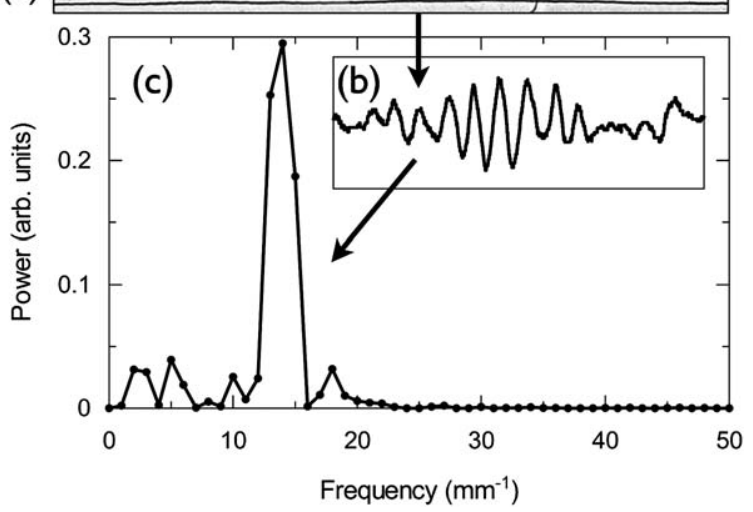

Fig. 2 Power spectrum for a wavy crack. Crack paths (a) were imaged, and the displacements (b) of the wavy cracks from the centre line between their two bounding cracks were extracted. The power spectrum (c) shows a single well-defined wavelength, which does not strongly depend on amplitude. There are no observable harmonics, suggesting that the crack path is close to sinusoidal, with a slowly varying envelope. The power spectra of the bounding, straight, cracks did not peak at any non-zero wavelength.

displacements of wavy cracks from the centreline were extracted, as shown in Fig. 2. A single wavelength could be identified for each wavy crack, with no harmonics.

Although wavy cracks in drying dispersions appear similar to the wavy cracks known from thermal quenching experiments on glass plates, ${ }^{3-8}$ the boundary conditions of the two cases are different. For a quenched plate all surfaces are free, whereas a drying film is normally fixed to a rigid substrate. Assuming no displacement on this interface, the thickness-averaged stress relieved by the presence of two parallel cracks at $y= \pm b$, in an elastic film, as shown in Fig. 3, is $\sigma_{c}=$ $\sigma_{0} \cosh (y c / h) / \cosh (b c / h)$ where $h$ is the film height, $\sigma_{0}$ is the local inplane pre-crack stress, and $c=0.9$ is a dimensionless decay length originating in the substrate constraint. ${ }^{12}$ The in-plane stresses between the two cracks, prior to the appearance of the wavy crack, are therefore $\sigma_{y y}=\sigma_{0}-\sigma_{c}$, and $\sigma_{x x}=\sigma_{0}-\nu \sigma_{c}$, where $\nu$ is Poisson's ratio of the film. This variation in stress, from the centreline to the bounding cracks, can guide the growth of the wavy crack.

The stresses in a drying film are a response to capillary forces. The magnitude of the capillary pressure in the film increases from zero, at the solidification front, to a maximum value at the pore-opening front. ${ }^{10}$ These fronts are separated by tens of crack spacings, as

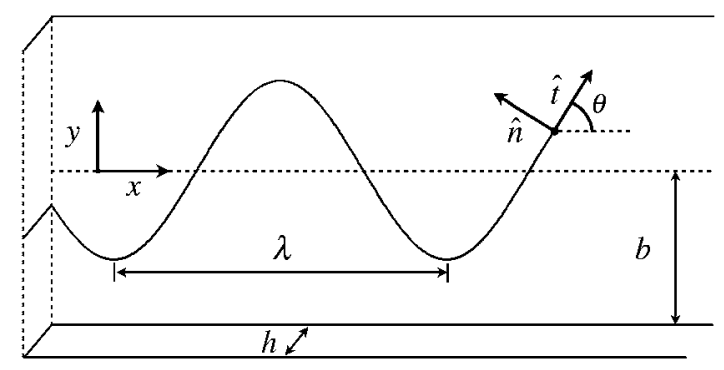

Fig. 3 Geometry of a wavy crack. A crack of wavelength $\lambda$ advances in the positive $x$-direction of a film of thickness $h$, with pre-existing cracks at $y= \pm b$. At each point there are unit vectors tangent $\hat{t}$ and normal $\hat{n}$ to the crack tip, rotated by an angle $\theta$ from the $x-y$ axes. demonstrated in Fig. 1(a), and the resulting stress gradients are correspondingly gentler than those generated by a wavy crack's bounding cracks. We therefore assume that there is always sufficient driving force to propagate the wavy crack quasi-statically, but that, otherwise, the stress gradients in the mean direction of crack growth are small. Within this gradient, the pre-crack stress, $\sigma_{0}$, at the wavy crack tip position is taken to satisfy the Griffith energy-balance condition.

Fracture mechanics in a plane can be consistently described ${ }^{1}$ in terms of either the stress intensity factors, $K_{I}$ and $K_{I I}$, which describe the variation of the opening and shear stress distributions near a crack tip, respectively, or a crack's strain energy release rate, $G$. These can take simple forms for a through-thickness crack in a thin film adhered to a rigid substrate, and are related by $G=\left(K_{I}^{2}+K_{I I}^{2}\right) / E$, where $E$ is the plane strain elastic modulus of the film. ${ }^{1}$ The stress intensity factors, and hence $G$, can be calculated by an integral formulation known as the weight function method, ${ }^{13,14}$ which accounts for the variations in pre-crack stresses along the path of the crack. The dominant length scale entering these calculations is the film thickness $h$, rather than the crack length, ${ }^{15}$ and the overwhelming contributions to $K_{I}$ and $K_{I I}$ arise from stresses acting within a distance $h$ from the crack tip. ${ }^{12}$ As shown in Fig. 4(a), the wavelengths of our wavy cracks were typically an order of magnitude larger than both the wave amplitudes, and the film thicknesses. The weight function method is simplified in this large-wavelength limit, and the tip of a wavy crack is well approximated by a straight crack, inclined at a small angle $\theta$ to the $x$-axis, with $K_{I}$ and $K_{I I}$ proportional to the prewavy-crack normal and shear tractions evaluated at the crack tip position $\vec{r}$. The leading term of the energy release rate of a wavy channel crack is then

$$
G=\frac{h}{c E}\left(\sigma_{y y}(\vec{r})^{2} \cos ^{2} \theta+\sigma_{x x}(\vec{r})^{2} \sin ^{2} \theta\right) .
$$

A general theory of path prediction should be expressible either in terms of the energy release rate of a crack, or the stress intensity factors around its tip. For a smooth in-plane crack whose path $\vec{r}(s)$ is parameterized by a path length $s$, there are tangent and normal unit vectors, $\hat{t}$, and $\hat{n}$ at each point along the path, as shown in Fig. 3. Here, as argued by Hodgdon and Sethna, ${ }^{16}$ the problem of path prediction simplifies to that of identifying a curvature function $A$ such that $d^{2} \vec{r} / d s^{2}=A \hat{n}$. In order to be independent of the choice of normal vector (which can be defined in either of two directions), $A$ must change $\operatorname{sign}^{16}$ under the transformation of $\hat{n}$ to $-\hat{n}$.

For a straight crack subject to uniform biaxial stress, the initial direction of crack growth ${ }^{17}$ is that which maximizes $G$. Path prediction in more complex situations has remained problematic. Often, the direction of maximum energy release rate coincides with the direction where the criterion of local symmetry, $K_{I I}=0$, holds. Much attention has therefore focused on the rigorous application of the criterion of local symmetry, which has been assumed to be maintained along the path taken. ${ }^{2,18-21}$ However, recent experiments have shown that wavy cracks in quenched glass, in fact, advance with $K_{I I} \neq 0 .{ }^{8}$ The two most well-developed modifications of local symmetry are that the curvature, $A$, is proportional to either $-K_{I I}$, or $-\nabla K_{I I} \hat{t}$; both models choose paths that attempt to minimize $\left|K_{I I}\right|$, and can also describe features of wavy cracks in thermally quenched glass plates. ${ }^{16,19-23}$ When applied to our system, however, they do not predict periodic paths, but rather cracks that curve directly to intersect with one of the bounding cracks, after any perturbation. Either additional physics is 

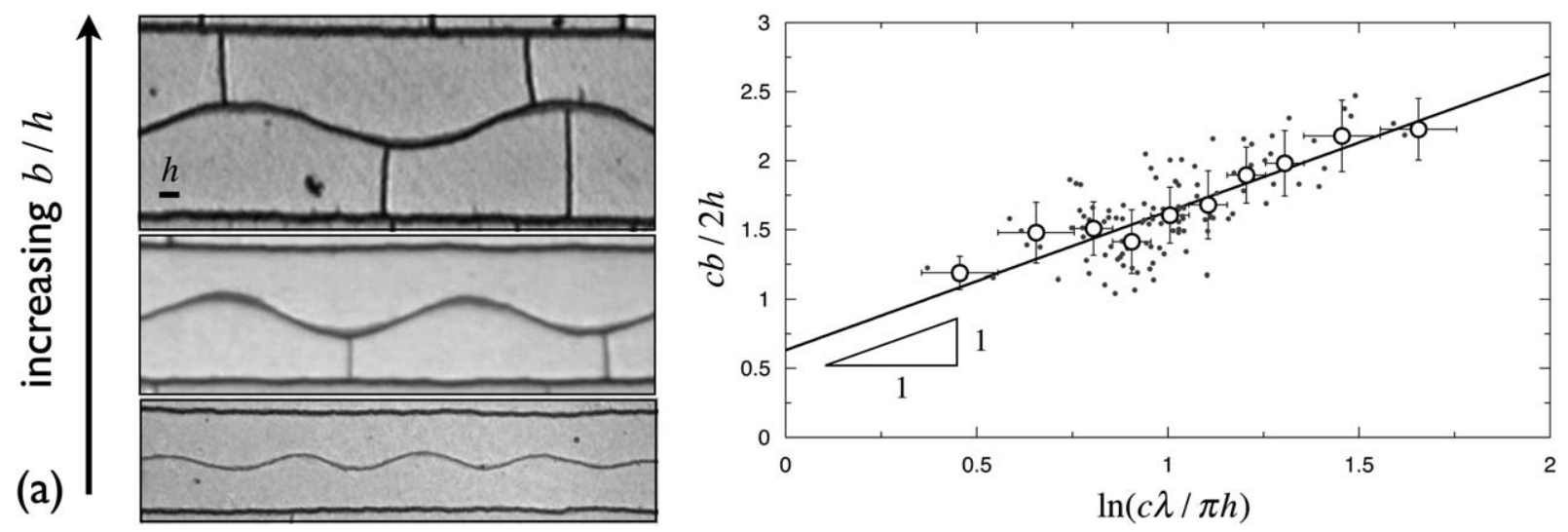

Fig. 4 Wavelength selection. (a) The wavelength depends on both the film thickness $h$, and the separation $b$ of the bounding cracks. For films of the same thickness (here $h=6 \pm 0.5 \mu \mathrm{m}$ ), the wavelength increases as $b / h$ increases. (b) Scaling of the wavelength. Raw data are shown as solid points, with binned data (open circles) highlighting the means and standard deviations of the observations. Along these axes, if the crack path is responding to gradients in energy release rate, eqn (4) predicts a slope of 1 , and an $y$-intercept of order 1 . A least-squares linear regression of the raw data finds a slope of $0.96 \pm 0.1$. The solid line shows the predicted slope of 1 , where the intercept $(0.64 \pm 0.03)$ alone has been fitted to the data by least-squares fitting.

needed to successfully model wavy channel cracks, or an alternate formulation is necessary. Following the latter approach, we here suggest a simple energy-based theory of crack paths, which can be applied broadly.

A crack path prediction may be constructed from the principle of maximum energy release rate. We argue that a crack will advance towards the direction that gives the greatest local increase in $G$, that is

$$
\frac{d^{2} \vec{r}}{d s^{2}}=\left(\frac{1}{\alpha} \nabla G \cdot \hat{n}\right) \hat{n},
$$

where $\alpha$ is a constant with dimensions of energy release rate. Under this proposition, an advancing crack will curve toward the direction that will release the most energy, with a curvature that is proportional to the local gradients in the energy release rate. More generally, a contribution to eqn (2) involving the direction of crack inclination, $\partial G / \partial \theta$, is allowed, but this will be vanishingly small in the longwavelength limit. This is equivalent to neglecting the shear stresses that may develop at an inclined crack tip.

For a crack travelling along the centreline the maximum energy release would be at an angle of $\pm 90^{\circ}$. This is because a crack normal to the boundary cracks releases the maximum component of $\sigma_{x x \text {. }}$ Indeed $G$, as defined in eqn (1), as a function of angle (for $y=0$ ) has a minimum in $G$ at $\theta=0$ and a maximum at $\pm 90^{\circ}$ However, as the crack deviates from the centreline it becomes closer to a boundary crack and therefore has less unrelieved stress to relax. This is seen in the function $G$ which has a maximum along $y=0$ and decreases monotonically for increasing magnitude of $y$. This moves the crack back towards the center, resulting in oscillatory motion. This is the origin of the waviness. A simple integration indicates that a sinusoid releases more energy per unit length than the straight crack. Quantifying this prediction involves applying the energy release rate derived in eqn (1) to the path prediction law given in eqn (2). Keeping only the leading order terms in the displacement $y$ of the crack tip from the centreline, and simplifying for the case $\exp (b c / h) \gg 1$, yields an equation for the motion of the crack tip

$$
\frac{d^{2} y}{d x^{2}}=-\left(\frac{4 G_{c} c^{2} e^{-c b / h}}{\alpha h^{2}}\right) y
$$

in the form of an undamped wave equation, where $G_{c}=h \sigma_{0}^{2} / c E$ is the fracture energy, or critical energy release rate. This predicts sinusoidal crack paths, with a wavelength $\lambda$ that depends on both the film thickness $h$, and the crack spacing $b$. In particular,

$$
\ln \left(\frac{\lambda c}{\pi h}\right)=\frac{1}{2} \ln \frac{\alpha}{G_{c}}+\frac{b c}{2 h}
$$

where $c$ is a known constant, and $b, h$, and $\lambda$ are experimentally observable length-scales.

To test our crack path model, the shapes of wavy cracks were observed in dried colloidal films. We observed the drying of a variety of dispersions: 8 mono-disperse preparations of colloidal polystyrene, with particle diameters between 100 and $400 \mathrm{~nm}$, and in three grades of Ludox, with particle diameters between 5 and $22 \mathrm{~nm}$. Wavy cracks were found in all materials, with no obvious superficial differences. Quantitative attention was then focussed onto a single dispersion, that of $100 \mathrm{~nm}$ polystyrene. The wavelength $\lambda$ and bounding crack separation $b$ of 119 individual wavy cracks were measured using an optical microscope. These cracks were observed in a set of films, with dried film thickness $h$ between 2 and $31 \mu \mathrm{m}$, as measured by scanning profilometry (Veeco Dektak 3M). Error estimates were made through repeated measurements on a subset of cracks, with average errors of $5 \%$ in the wavelength $\lambda, 3 \%$ in the spacing $b$, and $10 \%$ in the height $h$.

As the film thickness, $h$, increased, the average crack spacing and wavelength both increased. For cracks observed over a restricted range of $b / h$, for example between 3 and 3.5 , but over the full range of film thicknesses, there was no significant correlation between the scaled wavelength $\lambda / h$ and the film thickness (correlation coefficient $=$ $0.1)$. For wavy cracks observed at the same $h$, the wavelength depends on the separation $b$ of the bounding cracks, as demonstrated in Fig. 4 (a). Given the scatter in the data, it is difficult to distinguish between a linear and an exponential relationship. However, as shown in Fig. 4 (b), the data are in full agreement with the predicted wavelength scaling of smooth cracks advancing up gradients in $G$. Furthermore, the data suggest that the dimensionless group $\alpha / G_{c}$ is of order one, or that the unknown constant $\alpha$ in eqn (2) is proportional to, and approximately equal to, the fracture energy. 
Wavy crack patterns form from channel cracks in thin colloidal films under simple, repeatable conditions. Here, the leading-order predictions of existing crack path models do not predict periodic crack paths. Instead, the wavy cracks agree with crack paths that seek to maximize their energy release rate. We have shown how to adapt this principle into a law of crack path propagation, which is capable of describing the progress of a crack interacting with the complex stress states commonly encountered in engineering, or the physical and earth sciences.

The authors thank M. O'Sullivan for assistance in colloidal synthesis. Funding from the Engineering and Physical Sciences Council [EP/E05949X/1] is acknowledged.

\section{References}

1 B. R. Lawn, Fracture of Brittle Solids, Cambridge University Press, Cambridge, UK, 2nd edn, 1993, p. 378.

2 B. Cotterell and J. R. Rice, Int. J. Fract., 1980, 16, 155-169.

3 J. J. Hopfield, Nature, 1946, 158, 582-583.

4 A. Yuse and M. Sano, Nature, 1993, 362, 329-331.

5 O. Ronsin, F. Heslot and B. Perrin, Phys. Rev. Lett., 1995, 75, 2352-2355.

6 A. Yuse and M. Sano, Phys. D, 1997, 108, 365-378.

7 B. Yang and K. Ravi-Chandar, J. Mech. Phys. Solids, 2001, 49, 91-130.

8 K. Sakaue, S. Yoneyama and M. Takashi, Eng. Fract. Mech., 2009,

76, 2011-2024.
9 H. N. Yow, M. Goikoetxea, L. Goehring and A. F. Routh, J. Colloid Interface Sci., 2010, 352, 542-548.

10 R. C. Chiu, T. J. Garino and M. J. Cima, J. Am. Ceram. Soc., 1993, 76, 2257-64.

11 E. A. Jagla, Phys. Rev. E: Stat. Phys., Plasmas, Fluids, Relat. Interdiscip. Top., 2002, 65, 046147.

12 Z. C. Xia and J. W. Hutchinson, J. Mech. Phys. Solids, 2000, 48, $1107-1131$.

13 J. R. Rice, Int. J. Solids Struct., 1972, 8, 751-758.

14 T. Fett, C. Mattheck and D. Munz, Eng. Fract. Mech., 1987, 27, $697-$ 715.

15 J. L. Beuth Jr., Int. J. Solids Struct., 1992, 29, 1657-1675.

16 J. A. Hodgdon and J. P. Sethna, Phys. Rev. B: Condens. Matter, 1993, 47, 4831-4840.

17 F. Erdogan and G. C. Sih, J. Basic Eng., 1963, 85, 519527.

18 S.-I. Sasa, K. Sekimoto and H. Nakanishi, Phys. Rev. E: Stat. Phys., Plasmas, Fluids, Relat. Interdiscip. Top., 1994, 50, R1733-1736.

19 H.-A. Bahr, A. Gerbatsch, U. Bahr and H.-J. Weiss, Phys. Rev. E: Stat. Phys., Plasmas, Fluids, Relat. Interdiscip. Top., 1995, 52, 240243.

20 V.-B. Pham, H.-A. Bahr, U. Bahr, H. Balke and H.-J. Weiss, Phys. Rev. E: Stat., Nonlinear, Soft Matter Phys., 2008, 77, 066114.

21 F. Corson, M. Adda-Bedia, H. Henry and E. Katzav, Int. J. Fract., 2009, 158, 1-14.

22 M. Adda-Bedia and Y. Pomeau, Phys. Rev. E: Stat. Phys., Plasmas, Fluids, Relat. Interdiscip. Top., 1995, 52, 4105-4113.

23 E. Bouchbinder, H. G. E. Hentschel and I. Procaccia, Phys. Rev. E: Stat., Nonlinear, Soft Matter Phys., 2003, 68, 036601. 